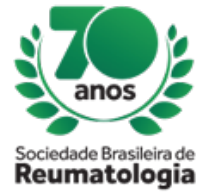

\title{
CUTANEOUS LESIONS IN PATIENT WITH JUVENILE SYSTEMIC ERITEMATOSUS LUPUS: HERPES ZOSTER X CITOMEGALOVIRUS
}

Paula Reale Fernandes (Universidade Federal de Juiz de Fora, Juiz de Fora, MG, Brasil), Ludmila Godinho Figueiredo (Universidade Federal de juiz de Fora, Juiz de Fora, MG, Brasil), Victor da Silva Coelho (Universidade Federal de Juiz de Fora, Juiz de Fora, MG, Brasil), Diana Campos Fernandino (Universidade Federal de Juiz de Fora, Juiz de Fora, MG, Brasil), Vania Schinzel (Universidade Federal de Juiz de Fora, Juiz de Fora, MG, Brasil)

\section{BACKGROUND}

Cytomegalovirus is a beta-herpesvirus commonly found in adolescents (30-70\%), most often asymptomatic or self-limited and may remain as a latent infection for years. It is thought that CMV infection is a possible trigger for the activation of the disease. Another hypothesis is that reactivation of latent infection secondary to immunosuppressive therapy occurs. The patient usually presents with persistent fever, anemia, lymphopenia, elevated liver enzymes. However, there may be other manifestations, more rare and potentially harmful. In these cases, remembering CMV as a possible diagnosis is crucial.

\section{CASE REPORT}

JSM, 12 years old, presenting a recent diagnosis of juvenile systemic lupus erythematosus, performing 3rd pulse therapy with methylprednisolone $1 \mathrm{~g}$ / day, 3 days, to control disease activity (alopecia, malar rash, arthritis, proteinuria, anemia and leukopenia). Stable from lupus disease, the following day presented erythematous vesicular lesions associated with local burning pain in the left iliac fossa region. She went to the medical service after 2 days of the onset of the disease, where she was diagnosed with herpes zoster and started treatment with acyclovir $400 \mathrm{mg}, 4$ times a day. After 1 day of treatment, the lesions, now pruritic and painless, spread to the rest of the abdomen, dorsal region and right cubital fossa, with no new symptoms. She was then hospitalized for differential diagnosis and increased dose of acyclovir to $500 \mathrm{mg}$ every 8 hours intravenously. No new symptoms appeared, only skin lesions. She remained hospitalized for 3 days and was discharged while still taking acyclovir, which she used for 10 days, with progressive improvement of the condition. Screening tests showed positive serology for cytomegalovirus $\lg M+/ \lg G-$

\section{CONCLUSION}

Cytomegalovirus in immunosuppressed patients has different presentations. Lupus-like symptoms are common, with persistent fever and cytopenias. Also described are more severe manifestations such as myocarditis and encephalitis. The disseminated cutaneous lesion is a rare but potentially fatal presentation. Early recognition and appropriate treatment of infection allows a favorable outcome for the patient. 